\author{
Anna Maziarczyk \\ Maria Curie-Skłodowska University \\ P1. M. Curie-Skłodowskiej 4a, \\ 20-031 Lublin, Poland
}

\title{
Les émotions de l'eau dans la littérature sentimentale. Le cycle de Marie de Jean-Philippe Toussaint
}

\begin{abstract}
The aim of this paper is to analyse diverse representations of water in Jean-Philippe Toussaint's cycle of Mary and the textual effects they produce. Water is not only the crucial aspect of the represented world but also the key narrative component, which shapes the plot, creates nostalgic atmosphere and reveals the philosophical dimension of the novel classifiable as romance. Drawing on selected theories of elements, the paper examines the ways in which Toussaint uses the emotions carried by water to create intensity and depth in a novel about love.

Keywords: cycle of Mary; water; emotions; philosophy
\end{abstract}

La création littéraire de Jean-Philippe Toussaint est puissamment élémentaire, surtout en ce qui concerne le cycle de Marie, tétralogie romanesque sur un amour difficile qui a particulièrement contribué à la renommée de l'auteur (Prix Médicis 2005, Prix Décembre 2009) et à sa popularité auprès des lecteurs. Les quatre éléments - composantes essentielles de l'univers et sa matière vivante selon les philosophes présocratiques - y sont largement thématisés et montrés dans une stricte corrélation avec l'existence humaine. L'axe de l'imagination élémentaire de l'écrivain est nettement fixé sur l'eau qui occupe une 
place privilégiée dans tous les volumes de la série. Abondamment évoquée dans ses diverses formes, naturelles et artificielles, géographiques, atmosphériques ou bien physiologiques, l'eau irrigue profondément la texture romanesque et la nourrit de façon stimulante sur plusieurs plans. Elle constitue non seulement l'élément préféré de l'écrivain, mais aussi une substance qui infuse de l'énergie romanesque à ses textes, comme il l'avoue lui-même dans une interview : «c'est une obsession, ça me plaît, c'est mon désir, c'est mon plaisir. J'ai toujours éprouvé du plaisir à parler de l'eau, à la décrire, à faire des métaphores aquatiques, fluides, liquides, mouillées » (Allemand 2011: 392). Le présent article se propose d'analyser diverses manifestations aquatiques dans le cycle de Marie et les effets romanesques qu'elles produisent. En nous référant à des travaux de réflexion sur les quatre éléments (philosophes grecs, Bachelard 1942, Durand 1985, Libis 1993), nous chercherons à démontrer la manière fine et complexe dont Toussaint exploite les émotions de l'eau pour donner de l'intensité et de la profondeur à l'histoire sentimentale qu'il raconte.

Sujet central de la tétralogie de Toussaint, la relation vertigineuse qui unit la créatrice de mode Marie Madeleine Marguerite de Montalte et le narrateur évolue sous le signe de l'eau, métaphoriquement ainsi que dans le sens littéral du terme. Se défaisant avant même de se faire réellement et se refaisant juste après, elle rappelle un cours capricieux et l'impétuosité d'un fleuve ou bien un ondoiement sans arrêt possible d'une mer, une fois calme et douce, une autre fois agitée à forte par des houles. Les protagonistes tantôt s'aiment éperdument, multiplient les gestes de tendresse et vivent des moments intimes d'une rare intensité, tantôt se séparent brusquement, tentent de se refaire une vie en solo et finissent par s'enfermer chacun dans sa solitude, souffrant d'un manque irrésistible de l'autre. Il y a, dans le dernier volume de la série, un long passage très émouvant où le narrateur, guettant en vain un coup de téléphone de son amie, ne cesse d'activer les souvenirs des moments passés ensemble afin de combler un vide émotionnel ne serait-ce que par l'imagination : 
[...] quand elle n'était pas là, Marie me manquait immédiatement, rien n'aiguisait autant mon amour pour elle que son éloignement - alors que dire de son absence ? [...] Marie [...] s'activait irrésistiblement dans mon esprit, sans que je fasse le moindre effort de conscience délibéré. [...] [J]e la laissais se mouvoir et s'animer dans mes pensées, tandis qu'elle se mettait à nager nue dans mes souvenirs ou à s'incarner dans les jardins de la propriété de son père. Je la revoyais alors dans le petit jardin de l'île d'Elbe, cette Marie dédoublée, ma Marie personnelle [...] (Toussaint $2013: 34$ ).

Les raisons de ces allées et venues sentimentales, de ces croissances et décadences alternées du sentiment restent largement obscures. Les ruptures surtout sont inexpliquées et totalement saugrenues : on ne sait pas ce qui cause la séparation définitive du couple, pourquoi le narrateur - contrairement à sa promesse - abandonne Marie dans un hôtel de Tokyo ni quelles sont les motivations de la créatrice de mode à chaque fois qu'elle disparaît de la vie de son ami sans donner quelque signe de vie. Les retours, en revanche, se produisent à chaque fois dans des conditions similaires: après la mort d'une personne proche, inattendue et subite, qui l'enlève à jamais et laisse un vide profond à l'intérieur. C'est pour le combler qu'on revient alors vers l'autre, amant(e) autrefois délaissé(e), en cherchant instinctivement du réconfort dans les bras du seul être cher qui reste encore au monde. Telles les vagues qui, après s'être écrasées sur des rochers, se brisent et rentrent vers le fond de la mer.

Cet amour houleux entre les protagonistes s'épanouit souvent au contact des éléments aquatiques: dans les cadres maritimes de la Méditerranée, dans l'atmosphère pluvieuse des métropoles où ils séjournent, sous l'effet des liquides absorbés d'une ou l'autre façon. Toussaint ne se satisfait toutefois pas, comme c'est fréquemment le cas en littérature, de faire naître de l'eau les flammes de la passion ${ }^{1}$, mais il réussit merveilleusement à harmoniser ce que Bachelard nomme « 'la matérialité' de certaines images de l'eau » (1942: 34) denses et évocatrices, encore qu'un tant soit peu clichés - avec divers aspects de la relation racontée. Ainsi, son côté romantique, avec tous

${ }^{1}$ Le motif des «eaux amoureuses » est largement analysé par Bachelard dans le premier chapitre de son fameux ouvrage L'eau et les rêves. 
les symptômes classiques tels le tressaillement d'émotions, l'émoi des sens, la délicatesse des gestes, est parfaitement ressorti dans la scène de la baignade dans la mer après l'enterrement du père de Marie. Structurée exactement comme dans des films à l'eau de rose - à la danse sur la plage enluminée par les rayons du soleil couchant suivent des jeux communs dans l'eau qui finissent par une plongée de Marie toute nue dans les vagues - elle dit de manière très touchante la proximité amoureuse qui renaît entre les amants séparés au contact de la nature et la consolation charnelle qu'elle apporte dans des moments difficiles. Ailleurs, plusieurs passages assez osés s'attachent à révéler la passion physique intense qui brûle entre le narrateur et Marie. Le désir sexuel s'éveille souvent sous l'impulsion d'un aphrodisiaque liquide, conventionnel comme le parfum ou l'alcool ou bien naturel comme l'eau de pluie ou du bain. Une fois encore, on remarque le caractère cliché de certaines scènes, par exemple celle de l'étreinte amoureuse sous l'effet du champagne semble activer quelque photo de Playboy ou être empruntée directement à un film érotique :

Quand elle eut fini de boire, elle tendit le bras au loin hors du lit pour déposer la flûte, qui tomba à la renverse sur la moquette, et, sans transition, d'un geste autoritaire, sûr et précis, elle s'empara de ma main et l'enfonça dans son slip, resserra les cuisses autour de sa prise. [...] [P]assé le premier saisissement, je sentis soudain sous la peau de mon doigt le contact légèrement électrique, éminemment vivant, meuble et humide, de l'intérieur de son sexe (Toussaint $2002: 28-29$ ).

Toussaint excelle d'ailleurs dans les représentations des corps mouillés, trempés par la pluie, rincés à l'eau courante, léchés par des vagues, transpirants de chaleur. De registres variés, elles montrent tout autant la beauté sensuelle et le charme féminin (Toussaint 2002: 166) que certains aspects de la physiologie humaine, pas forcément passionnants (sécrétions corporelles, liquides organiques). Cette précision et le goût du détail se manifestent à plusieurs reprises dans les descriptions des ébats amoureux qui prennent alors un ton impudique, indécent même, touchant au «porno soft », pour citer un critique (Claudel 2012 : 137). 
Tout en irriguant les émotions amoureuses qui composent l'intrigue, l'eau est également merveilleusement exploitée dans les quatre romans de la série pour créer des émotions romanesques. C'est largement elle qui les rend nostalgiques, tristes ou bien, selon les mots d'Houppermans, «imprégnées d'une profonde mélancolie » (2010). Car dans le cycle de Marie, l'univers tout entier est mouillé de pluie et de pleurs qui ruissellent à gros flots, presque sans arrêt, s'enchaînant l'un à l'autre et inondant doublement le paysage. Et l'eau, comme le souligne Bachelard à la suite d'Huysmans à qui il emprunte la formule, est «l'élément mélancolisant» par excellence (Bachelard 1942 : 123) qui engendre de l'ennui, des idées noires et toutes sortes d'états déprimants surtout en ce qui concerne ses deux manifestations mentionnées ci-dessus.

Les conditions atmosphériques dans lesquelles se joue l'histoire d'un amour compliqué s'harmonisent intimement et de façon plus que parfaite. À part une brève éclaircie ayant eu lieu sur l'Elbe après l'enterrement du père de Marie - qui s'est d'ailleurs particulièrement fort enracinée dans les souvenirs du narrateur comme le temps d'un bonheur lointain - l'eau coule du ciel constamment et sous diverses formes durant toute l'action. Tantôt abondantes, tantôt légères, les chutes de neige alternent avec une pluie verglaçante à Tokyo quand l'amour prend fin et les amants se séparent, il pleut à verse à Paris quand le nouvel amant y meurt subitement en pleine étreinte sexuelle, il y a du crachin durant le retour sur l'île après le décès de Maurizio, le gardien de la ferme familiale de Marie. Le récit abonde en images de villes sous la pluie et de pluie elle-même, peinte dans son impétuosité, ses bruits et les effets qu'elle produit sur le paysage environnant. Certaines sont de vraies «descriptions en dentelles » (Mannooretonil 2014 : 74), comme par exemple celle d'une averse sur Paris qui ouvre la période d'un rapprochement progressif des amants avant le dénouement heureux de l'histoire :

Au centre de la place, l'eau tombait en cascade le long des bassins à débordement de la fontaine Visconti, l'eau transparente, mobile, tourbillonnante, éclairée par des faisceaux de projecteurs blancs, qui dégringolait des vasques et bouillonnait dans le dernier bassin dans lequel la pluie continuait de tomber, mêlant l'eau à 
l'eau, tandis que les silhouettes imposantes des tours de Saint-Sulpice dressaient leurs profils mordorés en surplomb de la place (Toussaint $2013: 69$ ).

Inégalée dans sa précision de peindre la ville sous l'emprise de l'eau, la description oscille adroitement entre l'effet réaliste et une défamiliarisation à valeur métaphysique. Noyé dans la pluie qui submerge tout, traversé par les torrents d'eau qui s'écoulent massivement partout, Paris se voit imprégné d'une atmosphère maritime et se transforme en un océan invisible, masse informe et sombre, frémissante dans les ténèbres impénétrables. Ce parallèle aquatique entre le paysage urbain et la nature sert ici à évoquer le côté chaotique et fluctuant de la réalité, motif largement présent dans le cycle de Marie, tout en visant à la fois de monter la tension dramatique au moment de retrouvailles des protagonistes après une longue période de séparation.

Dans la plupart des cas pourtant, les conditions météorologiques sont présentées sous la forme de simples annotations du temps qu'il fait, assez neutres dans leur style sinon même réduites à des évidences dans la mesure où elles répètent des informations déjà fournies. Toussaint évite de décrire longuement la météorologie romanesque et préfère l'ébaucher par de toutes petites touches, ramenées à quelques phrases à peine, qui harmonisent bien avec cette forme aquatique considérée déjà par les Anciens comme la plus délicate, celle qui «surpasse[...] toutes les eaux en légèreté, douceur, ténuité et limpidité » (Hippocrate, $1804: 22$ ). La persistance du motif dans le texte et son déchiquetage ferme, en petites gouttes qui tombent ici et là dans le récit de sorte à illustrer de manière narrative le phénomène décrit, visent à maintenir les résonances mentales et symboliques de la pluie - tristesse infinie, ennui effroyable, solitude déchirante.

Il est difficile de constater si, dans le cycle de Marie, c'est la pluie qui entraîne des larmes ou bien si elles jaillissent toutes seules, de façon totalement indépendante. Sans aucun doute, elles ont des raisons de couler, le paysage pluvieux étant considéré par exemple par Bachelard comme la «matière du désespoir» ou bien le «néant substantiel » (Bachelard 1942: 113). En tout cas, on pleure abondamment dans ce récit, sans retenue et à toute occasion. Sans 
compter une ou deux scènes qui montrent le narrateur les larmes aux yeux, le privilège est nettement accordé à Marie qui sanglote aussi bien de douleur et d'émotion, quand tout s'écroule autour d'elle et que la réalité devient trop dure à supporter, que d'on ne sait pas trop quoi, dans des moments tout à fait inattendus, par exemple juste au début de sa relation avec le narrateur, «avant même qu'on s'embrasse pour la première fois » (Toussaint $2002:$ 12). La scène où l'on la voit pleurer en silence à l'hôtel de Tokyo, dans une étreinte amoureuse impossible qui signe la fin de l'amour, est une des plus touchantes dans la série et riche en significations tant symboliques que formelles ${ }^{2}$ :

[...] je vis apparaître très lentement une larme sous le mince rebord noir des lunettes de soie lilas, une larme immobile, à peine formée, qui tremblait tragiquement sur place, indécise, incapable de glisser davantage le long de sa joue, une larme qui, à force de trembler à la frontière du tissu, finit par éclater sur la peau de sa joue [...].

J'aurais pu boire cette larme à même sa joue, me laisser tomber sur son visage et la recueillir avec la langue. J'aurais pu me jeter sur elle pour embrasser ses joues, son visage et ses tempes, arracher ses lunettes de tissu, et la regarder dans les yeux [...], communier avec elle dans cette détresse que l'exacerbation de nos sens aiguisait, j'aurais pu forcer ses lèvres avec ma langue pour lui prouver la fougue de l'élan inentamé qui me portait vers elle, et nous nous serions sans doute perdus, en sueur, inconscients de nous-mêmes, dans une étreinte mouillée, salée, onctueuse, de baisers, de transpiration, de salive et de pleurs. Mais je n'ai rien fait [...]. J'ai regardé la larme se dissiper sur sa joue, et j'ai fermé les yeux - en pensant que peut-être, en effet, je ne l'aimais plus (Toussaint 2002 : 31-32).

Si Marie «ne pleure jamais à sens unique » (Blanckeman 2012 : 144), c'est non seulement parce qu'elle a une prédisposition naturelle à fondre en larmes au moindre stimulus, ce que le narrateur ne manque d'ailleurs pas de noter au passage, en faisant ressortir la symbolique de son prénom et les affinités avec la Madeleine biblique : «personne n'avait comme elle un tel talent lacrymal, ce don inné des larmes»

\footnotetext{
${ }^{2}$ La scène condense merveilleusement le caractère paradoxal de la relation amoureuse entre les protagonistes où la passion se heurte à des empêchements indéfinis, vagues mais toutefois particulièrement profonds. Placée au seuil de la série, elle rime par inversion avec le dénouement de l'intrigue.
} 
(Toussaint 2002: 55). En effet, Marie est un être aquatique, une véritable «fille de l'eau ( Dubois 2010), profondément liée à cet élément cosmique par toute sa nature. Elle en adore le contact physique, direct et total, qui la remplit d'un bonheur immense ou bien lui permet de retrouver un équilibre psychique après les traumatismes subis. On la voit souvent dans le texte se baigner dans la mer, toute nue, appréciant les caresses des vagues sur son corps ou se hasardant sans crainte vers les fonds maritimes. Cette fascination la marque entièrement jusque dans sa vie professionnelle: créatrice de mode, elle s'inspire dans son art de la nature environnante pour inventer des collections spectaculaires de robes d'eau sous diverses formes, flottantes comme celle en gorgone de mer ou fondantes comme celle en sorbet, avec un joyau incontestable du catalogue - la robe en miel, « une robe en lévitation, légère, fluide, fondante, lentement liquide et sirupeuse, en apesanteur dans l'espace et au plus près du corps du modèle, puisque le corps du modèle était la robe elle-même » (Toussaint $2013: 12$ ).

Mais surtout, Marie est, elle-même, comme l'eau : imprévisible et instable dans ses réactions, chaotique de nature avec sa tendance à accumuler les choses en les laissant traîner partout et pleine d'un charme individuel qu'elle répand sur tout ce qui l'entoure. Jacques Dubois a bien raison de constater que « la liquidité de Marie se traduit donc en expansion de soi et de tout ce qui la prolonge » (Dubois 2010), mais il y a plus que ça. Tout comme l'eau s'adapte à la forme du récipient qu'elle remplit, Marie s'adapte au monde d'alentour, elle ressent intuitivement sa pulsion, sa tonalité, ses modulations et y répond par des émotions et des comportements similaires, donnant à l'occasion l'impression d'être une femme capricieuse et quelque peu réfractaire aux normes sociales. Cette faculté toute particulière, largement connue en psychologie ${ }^{3}$, est dans le texte désignée par la

\footnotetext{
${ }^{3}$ Les sciences psychologiques, notamment la psychanalyse de Freud, renvoient au concept de «sentiment océanique » formulé par Romain Rolland, par lequel on désigne une sensation d'unité avec l'univers éprouvée ou désirée par l'individu, pour débattre sur les origines du besoin religieux chez les gens.
} 
formule de «disposition océanique » qui déforme quelque peu la dénomination scientifique en vigueur pour démontrer par là même l'intensité très élevée avec laquelle elle se manifeste dans le cas de Marie :

Marie avait ce don, cette capacité singulière, cette faculté miraculeuse, de parvenir, dans l'instant, à ne faire qu'un avec le monde, de connaître l'harmonie entre soi et l'univers, dans une dissolution absolue de sa propre conscience. [...] Marie atteignait d'instinct la dimension cosmique de l'existence [...] (Toussaint $2013: 37)$.

La symbiose entre Marie et le monde, qui se réalise essentiellement sur un mode aquatique, aiguise encore davantage le ton mélancolique et rêveur du cycle. Elle incite à réfléchir sur les sujets séculaires tels que l'existence de l'homme et sa petitesse face à l'infini de l'univers, tout comme sur diverses façons de vivre cette vie minuscule.

L'on voit là, dans ce ton réflexif, se profiler encore un enjeu du motif aquatique dans la série étudiée, symbolique sinon même métaphysique. Si abondamment évoquée dans le texte, l'eau est chargée de générer des significations implicites qui enrichissent considérablement l'intrigue amoureuse. Ce n'est pas sans raison que Toussaint place majoritairement l'action romanesque dans des cadres aquatiques : outre les métropoles noyées sous des pluies diluviennes, elle se déroule dans une large part au bord de la Méditerranée. Car la mer, espace intermédiaire entre le fleuve et l'océan, en réunit les propriétés de sorte à représenter de façon idéale l'eau dans son essence même et dans sa profonde ambiguïté. Depuis toujours, l'eau a été le symbole d'un élément puissant où se conjuguent les forces contradictoires de la vie et de la mort. Cette ambivalence est parfaitement mise en valeur par Toussaint de sorte à apporter au récit une touche quelque peu philosophique.

À plusieurs reprises, les images de l'eau véhiculées dans le texte engendrent un malaise indistinct, voire même une peur immense devant les dangers concrets et physiques, liés à sa force destructrice contre laquelle l'homme ne peut rien et face aux menaces à caractère existentiel qu'elle représente. Le contact de l'individu avec l'élément liquide ne se fait pas sans risques: le père de Marie, habitué de la 
Méditerranée au bord de laquelle il passe une large part de sa vie, meurt en se noyant on ne sait pas trop dans quelles conditions et la créatrice de mode, elle-même, faillit être emportée par des vagues dans les «zones huileuses, noires et denses, impénétrables » (Toussaint 2005: 183) de la mer quand, frappée par le deuil, elle plonge la nuit dans les eaux ténébreuses. Ce comportement de l'hérö̈ne n'est pas sans rappeler ce que Bachelard désigne par le « complexe d'Ophélie » (Bachelard 1973: 112), à savoir une aspiration suicidaire à se perdre dans les flots maritimes, une volonté de précipiter sa fin en s'y diluant. Impossible de ne pas remarquer la complexité de l'actualisation de ce complexe par Toussaint : l'acte de Marie est symétrique au sort de son père, comme si elle voulait plutôt poursuivre le défunt que simplement s'anéantir, et il ressemble à un défi existentiel au destin dans la mesure où l'eau, élément intimement lié à la personnalité de la femme, est amenée à décider de son sort. $\mathrm{Si}$, dans le cycle analysé, l'eau ne met pas définitivement un terme à la vie humaine sur le plan de la diégésis, elle l'évoque de diverses manières. Les manifestations aquatiques servent souvent à annoncer les événements dramatiques de l'intrigue ou des catastrophes, comme c'est, par exemple, le cas d'une longue description des aspects étranges de la crique habituelle des protagonistes qui, terminée par une phrase on ne peut pas plus explicite - «Nous étions sortis de l'eau, et nous nous faisions sécher sur les rochers, regardant la mer grise qui clapotait devant nous dans cette atmosphère de fin du monde » (Toussaint 2009 : 186) - précède l'épisode de l'incendie fatal sur l'île d'Elbe qui ravage la ferme familiale de Marie avec ses chevaux. Ailleurs, elles ne font que susciter des connotations liées au champ sémantique de la mort, plus ou moins motivées par l'intrigue. L'été des funérailles du père de Marie, la Méditerranée est tranquille, plate, sans le moindre clapotis de surface et fait penser à un lieu de repos éternel ou bien à un cercueil naturel, idéal, nettement plus approprié à l'homme que le tombeau conventionnel. Cette immobilité sépulcrale caractérise également l'eau de la piscine sur le toit de l'hôtel à Tokyo ou bien celle d'un bain préparé par Marie, ce qui apporte une touche quelque peu métaphysique à des scènes qui, en 
apparence, n'ont rien à voir avec la problématique des questions ultimes. L'eau, dit Gilbert Durand, est « une grande épiphanie de la Mort » (1984: 104) et cette conviction revient avec insistance dans les réflexions de Bachelard, Libis ou autres chercheurs qui s'attachent à établir l'imaginaire culturel lié à cet élément et analyser ses diverses hypostases. Il en est exactement de même chez Toussaint : l'eau y est largement thanatomorphe, associée à l'idée de la mort, de l'achèvement inévitable, de la perte et de la destruction. Pure et immense, elle représente par opposition le côté sombre de l'existence humaine : sa finitude.

Or, dans le cycle de Marie, l'eau est également montrée dans sa valence positive, comme source d'énergie vitale, de réel bonheur ressenti de manière sensuelle, de profonde régénération physique et mentale. À chaque fois qu'elle s'ébat à la plage ou plonge dans les vagues, Marie paraît heureuse comme jamais, comme à aucun autre moment de sa vie, y compris ceux où elle fait l'amour. L'eau a sur elle une influence visiblement bienfaisante et c'est vers la mer qu'elle dirige ses pas après la mort de son père et celle de son amant JeanChristophe de G., cherchant de la consolation dans le spectacle des grandes marées et à leur contact direct. Car la mer qui, selon le poète, « offre à la fois l'idée de l'immensité et du mouvement » (Baudelaire 1980 : 418), lui permet d'accoler sa finitude humaine à l'infini et à l'éternel de la nature. Dans une contemplation muette de l'élément puissant ou une compétition risquée avec ses forces, il lui devient possible - rien que par le rapprochement au plus près de cette énergie et violence naturelles - de retrouver ses propres forces vitales, d'accepter le cours des choses et vaincre l'angoisse de l'inévitable. Il y a d'ailleurs, dans le texte, une scène spectaculaire où le narrateur se baigne dans une piscine étoilée sur le toit d'un hôtel japonais et qui laisse voir la puissance extraordinaire de l'eau sur l'homme, sa capacité phénoménale de le libérer pas seulement des soucis psychiques et des fatigues physiques, mais aussi de toutes ses limitations quelle que soit leur nature :

J'avais le sentiment de nager au cœur même de l'univers, parmi des galaxies presque palpables. [...] Je nageais comme en apesanteur dans le ciel, respirant 
Les émotions de l'eau dans la littérature sentimentale. Le cycle de ... 173

doucement en laissant mes pensées se fondre dans l'harmonie de l'univers. J'avais fini par me déprendre de moi, mes pensées procédaient de l'eau qui m'entourait, elles en étaient l'émanation, elles en avaient l'évidence et la fluidité, elles s'écoulaient au gré du temps qui passe et coulaient sans objet dans l'ivresse de leur simple écoulement [...] et je pensais, mais c'est déjà trop dire, non, je ne pensais pas, je faisais maintenant corps avec l'infini des pensées, j'étais moimême le mouvement de la pensée, j'étais le cours du temps (Toussaint 2002 : 51$52)$.

À associer cette scène à celle citée au début de notre article, qui montre le narrateur regarder la pluie dégouliner sur Paris et faire revivre Marie dans ses rêveries, on peut découvrir le sens de l'hydrographie toussaintienne. Évoluant entre deux pôles opposés, l'eau létale qui porte la mort, noire, agressive, meurtrière et l'eau lustrale qui, à en citer Eliade, affiche la «vertu de purification, de régénération et de renaissance »(Eliade 1996: 170), elle est censée ressortir moins la bivalence de l'élément aquatique qu'illustrer sa profonde instabilité, un mouvement infini et incontournable qui la caractérise indépendamment de ses formes. La symbolique nostalgique de telles images aquatiques est parfaitement connue: «L'eau qui coule est la figure de l'irrévocable » affirme Durand (1985 : 104), en paraphrasant la célèbre réflexion d'Héraclite "panta rei » qui dit le mouvement irréversible du temps en le comparant au courant du fleuve ( $c f$. Héraclite $2006: 24)$. C'est justement ce sens qui se dégage des scènes de bains, de pluie, de pleurs particulièrement nombreuses dans les quatre romans : la liquidité y dit la fluidité des sentiments qui ne durent pas et celle des choses en perpétuelle transformation. Véhiculé essentiellement à travers les manifestations aquatiques, outre, bien évidemment, l'intrigue romanesque elle-même qui le thématise, le motif central de la série est également accentué par d'autres images suggestives de l'écoulement des vies, de l'impermanence des êtres et de l'éphémère des choses. Rappelons ici ne seraient-ce que les multiples évocations des phénomènes évanescents tels que les vacillements des ombres, les vibrations des sons, les jeux des lumières, les descriptions des instants temporels transitoires au croisement du jour et de la nuit ou bien les représentations du mouvement même dans les scènes des 
déambulations à travers les villes et les voyages ferroviaires, aériens et maritimes largement racontés. Parmi toutes ces formes romanesques du motif de la fluidité universelle, les manifestations aquatiques sont, sans doute, particulièrement expressives. L'eau qui coule du ciel et des yeux, qui circule en permanence à la surface de la terre, qui s'agite dans une mer tantôt démontée et tantôt calme, enfin cette eau qui transforme l'homme métaphorise de façon on ne peut plus parlante le temps qui passe et les changements de la vie.

Si l'aquatique dans le cycle de Marie joue dans une large part «sur un mode dysphorique » (Wagner 2010), engendrant l'angoisse métaphysique d'un flux temporel irrévocable, il est également la clé d'une expérience de transcendance permettant à l'homme de se libérer de l'emprise du temps. Car l'eau, comme d'ailleurs l'ont montré Bachelard, Durand et d'autres chercheurs, possède un extraordinaire potentiel à nourrir l'imagination, réveiller des rêveries et enrichir ainsi la vie de l'individu par une expérience des réalités illusoires, inventées mentalement. Toussaint, quant à lui, la montre comme un stimulus idéal non pour la création de la nouveauté mais pour la recréation du passé qui s'en est allé à jamais. Indépendamment de ses manifestations, l'eau coïncide souvent dans sa tétralogie avec la rêverie, tantôt inopinée et rapide, tantôt intentionnelle et prolongée dont on se refuse de sortir. À plusieurs reprises, on voit les personnages plonger dans des souvenirs, revivre les moments d'autrefois ou tout simplement s'adonner à des méditations silencieuses en regardant la pluie tomber ou les vagues de la mer s'agiter dans leur mouvement infini. C'est surtout le temps pluvieux qui incite à l'imagination et à la réflexion : quand les contours de la réalité se diluent dans les eaux qui, à en citer Eliade, «désintègrent, abolissent les formes » (Eliade 1996 : 173), ceux de l'ancien temps se reprofilent dans la mémoire doucement, de plus en plus nets, faisant réapparaître, simultanément, les visages des gens qui ne sont plus là. Car le monde qui disparaît sous le voile de la pluie rend la disparition des êtres chers d'autant plus poignante et le processus de la reconstruction du passé s'active de manière naturelle. Nous avons déjà vu la scène où le narrateur, se retrouvant seul dans un Paris noyé sous 
Les émotions de l'eau dans la littérature sentimentale. Le cycle de ... 175

des trombes d'eau après un été passé à deux sur l'île d'Elbe ensoleillée, ne cesse de se rappeler les petits instants de bonheur qu'il y a vécus et les diverses effigies de sa bien-aimée: Marie dans le jardin, dans sa chambre à l'étage, à la plage. Le troisième volume de la série, La Vérité sur Marie, est presque entièrement basé sur ce mécanisme : le récit n'est pas une simple chronique des faits, neutre et objective, mais plutôt leur reconstruction mentale et hypothétique selon le narrateur momentanément hétérodiégétique. Focalisé sur l'épisode de la rupture définitive du couple, il laisse voir la vie de la créatrice de mode après la séparation à Tokyo telle que le narrateur se l'imagine s'être déroulée. Ses visions se nourrissent en partie des faits qu'il a pu connaître pour ainsi dire de première main, par les relations sommaires de Marie, mais elles restent dans une large mesure purement imaginaires, fondées sur ses projections, suppositions ou fantasmes, le récit étant «sujet à l'erreur ou à la fabulation» (Richir 2013 : 65). Les scènes de rêveries sont d'ailleurs les plus belles de la tétralogie, d'une délicatesse et d'une sensualité inouïes, et en disent long surles sinuosités du sentiment qui s'épanouit paradoxalement dans l'éloignement, quand l'être aimé n'est pas là. Progressivement, on découvre la puissance sensationnelle de ces moments mélancoliques où le narrateur non seulement retrouve la réalité passée, mais la transfère vers le présent, en superposant l'une sur l'autre les deux dimensions temporelles pour vivre simultanément toutes ses vies à la fois, dans une sorte de synchronisme total :

C'est quand on se promène dans le temps, et qu'on a la sensation d'être à la fois dans le présent et dans le passé - parce que les différents moments du temps ne sont plus hiérarchisés par le souvenir - que l'esprit peine à ajuster ses repères, parce que le temps, alors, n'est plus perçu comme la succession d'instants qu'il a toujours été, mais comme une superposition de présents simultanés (Toussaint 2013).

Voilà le temps aboli et l'éternité atteinte. Le passé, ancré dans le présent par un mouvement d'imagination, cesse de se dissiper en miettes et forme avec le présent une réalité pluridimensionnelle, cosmique, absolue. Les rêveries que l'eau seule est capable d'activer 
multiplient ainsi à l'infini l'intensité de l'existence et plus que ça : elles rendent possible une expérience de l'infini.

Comme de nombreux artistes et chercheurs, Toussaint cède à l'envoûtement de l'élément liquide ; qui plus est, il en fait le principe de sa poétique romanesque. Dans le cycle de Marie, l'eau n'est pas materia primordialis du texte, mais plutôt son support inhérent qui œuvre sur plusieurs plans de sorte à le laisser s'épanouir dans sa densité et déplier de nouvelles strates de sens. Elle imprègne, tout d'abord, l'intrigue passionnelle et la rend d'autant plus passionnante, faisant ressortir divers aspects de la relation entre Marie Madeleine Marguerite de Montalte et le narrateur : du romantique à l'érotique, en frôlant un tant soit peu le pornographique. C'est elle aussi qui donne un charme particulier à la série romanesque, en créant une ambiance mélancolique, teintée de tristesse, de nostalgie, d'anxiété et de songe, une sorte de spleen poétique qui rime bien avec le récit de l'amour impossible et désiré, renaissant de ses propres naufrages. Elle joue enfin sur le plan symbolique, enrichissant une histoire somme toute banale d'une réflexion métaphysique sur l'univers et la vie, sur l'éphémère des choses et des êtres. "Plus qu'un autre élément peutêtre, l'eau est une réalité poétique complète » dit Bachelard (1942: 21) et c'est parfaitement visible dans le cycle de Marie où cette poésie est merveilleusement rendue et mise au profit d'une fiction romanesque, subtile et profonde à la fois.

\section{Bibliographie}

Bachelard, G. (1942) : L'eau et les rêves. Essai sur l'imagination de la matière. Paris : José Corti.

Baudelaire, Ch. (1980) : Euvres complètes. Paris : Laffont Bouquins.

Blanckeman, B. (2012) : Faire l'amour à la Toussaint (sur quelques postures littéraires minimalistes. In : M. Dambre, B. Blanckeman (dir.), Romanciers minimalistes 1979-2003. Paris : Presses Sorbonne Nouvelle, pp. 141-153.

Claudel, Ph. (2012) : Faire (ou défaire) l'amour : géographie de l'éros dépité. In : M. Dambre, B. Blanckeman (dir.), Romanciers minimalistes 1979-2003. Paris: Presses Sorbonne Nouvelle, pp. 127-139.

Dubois, J. (2010) : Avec Marie. Textyles, 38, pp. 13-23.

Durand, G. (1985) : Les structures anthropologiques de l'imaginaire. Paris : Dunod. 
Les émotions de l'eau dans la littérature sentimentale. Le cycle de ... 177

Eliade, M. (1996) : Traité d'histoire des religions. Paris : Payot.

Héraclite (2006) : Fragments. Genève : Arbre d'Or.

Hippocrate (1804): Traité des airs, des eaux et des lieux. Paris: Croullebois, Bossange, Masson et Besson. Version disponible en ligne: https://books.google.pl/books?id=2TYdA0j8WaYC\&printsec=frontcover\&source =gbs_book_other_versions_r\&redir_esc $=\mathrm{y} \# \mathrm{v}=$ onepage $\& \mathrm{q} \& \mathrm{f}=$ false, $\quad$ page consultée le 11 octobre 2017.

Houppermans, S. (2010) : L'autre fugitive. Textyles, 38, pp. 109-120.

Libis, J. (1993) : L'eau et la mort. Dijon : Éditions universitaires de Dijon.

Mannooretonil, A. (2014) : Jean-Philippe Toussaint ou l'art délicat de l'infinitésimal. L'art et littérature, 4208, pp. 73-82.

Richir, A. (2013) : Fantasmer la vérité sur Marie. Cahiers ERTA, dossier Déformer le réel, 3, pp. 53-66.

Toussaint, J.-Ph. (2002) : Faire l'amour. Paris : Les Éditions de Minuit.

Toussaint, J.-Ph. (2005) : Fuir. Paris : Les Éditions de Minuit.

Toussaint, J.-Ph. (2009) : La vérité sur Marie. Paris : Les Éditions de Minuit.

Toussaint, J.-Ph. (2013) : Nue. Paris : Les Éditions de Minuit.

Wagner, F. (2010) : Monsieur Jean-Philippe Toussaint et la notion de vérité. Textyles, 38, pp. 25-34. 